\title{
Y-maze avoidance: an automated and rapid associative learning paradigm in zebrafish
}

\author{
Ryo Aoki ${ }^{1,2}$, Takashi Tsuboi ${ }^{2}$ and Hitoshi Okamoto ${ }^{1, \S}$
}

${ }^{1}$ Laboratory for Developmental Gene Regulation, Neural Growth and Regeneration Research Group, RIKEN Brain Science Institute, 2-1 Hirosawa, Wako, Saitama 351-0198, Japan

${ }^{2}$ Department of Life Science, Graduate School of Arts and Sciences, The University of Tokyo, 3-8-1, Komaba, Meguro, Tokyo 153-8902, Japan

§Correspondences should be addressed to Dr. Hitoshi Okamoto

Hitoshi Okamoto, M.D., Ph.D.

Laboratory for Developmental Gene Regulation

Neural Growth and Regeneration Research Group

Brain Science Institute, RIKEN

2-1 Hirosawa, Wako, Saitama 351-0198, Japan

Tel: +81-48-467-9712; Fax: +81-48-467-9714

E-mail: hitoshi@brain.riken.jp

11 pages and 2 figures are submitted.

Figure 1 should be printed in color. 


\begin{abstract}
Recent genetic and neuroanatomical studies have demonstrated the suitability of adult zebrafish as a model animal in behavioral neuroscience. To evaluate their ability for adaptive learning, it is beneficial to develop a more efficient, more precise, and less laborious behavioral paradigm. By combining real-time video tracking and computer-controlled visual cue presentations on a liquid crystal display screen under the tank, we have developed a new method by which zebrafish can be trained to avoid one arm of a Y-shaped tank by presenting a specific color on the floor paired with a noxious electric shock. The whole procedure takes less than 2 hours, and zebrafish learn to choose the correct arm of the tank at an efficiency rate of $89.0 \%$, which is high compared with other associative learning paradigms. In addition, the acquired memory lasted for 24 hours. We also developed a graphical user interface by which users can modify the paradigm assessment parameters such as shape of the tank and time schedules. Our assay system enables the rapid and reliable evaluation of cognitive ability in adult zebrafish, with high reproducibility, minimal experimenters' bias, and ease of customization.
\end{abstract}

Keywords: Adult zebrafish, Aversive learning paradigm, MATLAB, Automated position detection 


\section{Introduction}

Despite the widespread study of zebrafish in fields such as developmental biology, little attention has been paid to this important animal model in terms of behavioral neuroscience due to the lack of knowledge about their neural circuitry. However, recent studies of comparative neuroanatomy and lesion experiments have supported the idea that telencephalic brain regions responsible for adaptive behaviors in mammals such as cortex, hippocampus, and basal ganglia are evolutionarily conserved in teleost species (Mueller and Wullimann, 2009; Portavella et al., 2004; Rodríguez et al., 2002), suggesting the potential value of zebrafish as a model animal in behavioral and cognitive neuroscience. In addition, the wide variety of genetic techniques available in zebrafish such as selective gene introduction (e.g., BAC transgenesis (Higashijima et al., 2004), enhancer trapping (Kawakami et al., 2004)) and gene targeting (Meng et al., 2008) now make it possible to clarify the neural circuitry and mechanisms commonly used from teleosts to mammals to regulate adaptive behaviors.

Several paradigms have already been developed to measure the capacity for adaptive learning in zebrafish, including active avoidance (Aoki et al., 2013; Portavella et al., 2004; Pradel et al., 1999), T-mazes (Darland and Dowling, 2001), delayed spatial alteration (Levin and Chen, n.d.), plus-mazes (Al-Imari and Gerlai, 2008; Sison and Gerlai, 2010), and conditioned place preference (Darland and Dowling, 2001; Lau et al., 2006). A reward-based automated visual choice discrimination paradigm was also reported recently (Mueller and Neuhauss, 2012). However, all these methods are very timeconsuming due to their heavy reliance on manual experimentation. In addition, the reported protocols have to be modified for each different functional analysis of an adaptive behavior, and it is laborious to rebuild such paradigms from the beginning.

We therefore developed a rapid, flexible, and fully automated Y-maze avoidance task as a novel paradigm for adaptive learning. Our task offers reliable measurement of the capacity for associative learning and also dramatically increases productivity of the experiment. Moreover, our task uses an inexpensive and open source code for the experimental operation, enabling each researcher to easily recapitulate and modify the paradigm to examine their function of interest. 


\section{Materials and Methods}

\section{Animals}

Zebrafish (Danio rerio) were bred and raised under standard conditions (Westerfield, 2007). Prior to the experiments, fish were raised and maintained in $7-1$ tanks at $28^{\circ} \mathrm{C}$, with continuous water exchange under standard 14-h light/10-h dark cycling. All protocols were reviewed and approved by the Animal Care and Use Committees of the RIKEN Brain Science Institute.

\section{Training apparatus}

Our setup consists of a 24.1" LCD screen (MultiSync LCD-P232W, NEC, Japan) in a soundproof box and a Y-shaped tank with three identical arms (height $=10 \mathrm{~cm}$, length $=15 \mathrm{~cm}$, width $=7 \mathrm{~cm}$ ) and a translucent floor, filled with water (depth $=8 \mathrm{~cm}$ ) and placed on the screen (Figure 1A). A video camera for recording the fish behaviors (HD Pro Webcam C920t, Logicool, Japan) is placed 60 $\mathrm{cm}$ above the tank and connected to a personal computer, which controls the presentation of visual cues on the screen and tones via a speaker. Electric shocks were generated by bath amplifier (SEG3104, Nihon Koden, Japan) controlled by a stimulator (master-8, A.M.P.I., Israel)(DC), or volt slider (v-130-3, Yamabishi electric co., ltd., Japan) controlled by I/O device (NI USB-6501, National Instruments, Texas)(AC). Electric shocks (DC: $5 \mathrm{~V}(0.12 \mathrm{~mA}), 50-\mathrm{ms}$ duration, $10 \mathrm{~Hz}, \mathrm{AC}: 2.5 \mathrm{~V})$ are delivered via steel mesh plates placed on both sides of the three arms. Small fans are also put on the screen to dissipate the heat generated by the screen and keep the water temperature at $28-30^{\circ} \mathrm{C}$.

\section{Software algorithm}

Our custom-written MATLAB source code for the Y-maze paradigm is described in supplementary toolbox 1 as Ymaze_discrimination. Before putting the fish into the tank, the positions of 13 regions of interest (ROIs) (1-3: distal part; 4-6: middle part; 7-9: proximal part of the three arms, respectively; 10: central triangle region; 11: circular marker indicating the current running program (trial or ITI); 12: circular marker indicating that electric shock is on; 13: unused circular marker) are assigned on the camera frame. To detect the fish position, the image captured by the camera is first transformed into grayscale then subtracted from the reference image. Because the zebrafish looks darker than the background, the subtracted image, after eliminating noise, indicates the fish location. The reference image is renewed each time the image presented on the floor is changed. We manually checked and confirmed that the assignment of the fish position to each ROI was completely correct. Our graphical user interface (GUI)-based MATLAB source code is also attached as custom_maze (Supplementary figure 1). 


\section{Behavioral training}

We used wild-type adult zebrafish, 6-12 months old, which were separated from other fish and placed in individual 1-1 tanks one day prior to the start of experimentation. On the training day, each fish was placed in the Y-maze tank with a transparent lid to prevent escape, and habituated to the training apparatus for 10 min before starting the trials. During this time, the floor of all regions of the tank was colored gray (53 lux). The location of the fish in the Y-maze tank was continuously monitored by the video camera placed above the tank (Figure 1A). The training procedure consisted of an initial 20 'no shock' trials and subsequent 100 'shock' trials. At the beginning of each trial, an auditory cue $(700 \mathrm{~Hz}, 0.5 \mathrm{sec}, 63 \mathrm{~dB})$ was presented. Then, one arm end (within $10 \mathrm{~cm}$ from the distal edge) in which the fish was neither present nor last visited was randomly chosen as a goal region, and all floors except that region were changed to red (27 lux) using the LCD screen placed under the tank (Figure 1B). This color cue presentation was maintained until the fish reached the goal region, or until 30 seconds passed. If the fish reached the goal region, an auditory cue $(1400 \mathrm{~Hz}$, $0.5 \mathrm{sec}, 63 \mathrm{~dB}$ ) was given. In the 'shock' trials, if the fish did not reach the goal region within 15 seconds, all regions within the tank except the goal arm received the continuous electric shock until the trial finished. For all trials, the arm the fish visited first and the latency to reach the goal region were recorded. During 30 seconds of the inter-trial intervals, the entire floor was colored gray. 


\section{Results}

\section{Rapid acquisition and accurate performance of learner fish}

A typical example of fish behavior during training is shown in Figure 2A; the zebrafish represented was very active in locomotion and never stayed at one arm. Furthermore, in all 'no shock' trials in which the electric shock was absent, it reached the goal region within 15 sec. However, there was no obvious tendency to visit the goal arm first rather than the non-goal arms, suggesting that there was no preference based on floor color. On the contrary, in the 'shock' trials, the correct rate (calculated as an average of 10 trials) quickly increased to reach $100 \%$ by trial $41-43$. Surprisingly, at that time, the fish had experienced the electric shock only three times (Supplementary Movie 1). Subsequently, the success in reaching the goal region decreased despite the fish receiving the electric shock repeatedly (trials 44-61). During this period, the fish was often observed freezing until the electric shock was turned on, then immediately after the electric stimulus onset, it rushed into the goal region with a burst swim. During trials 62-120, the fish success rate recovered and stabilized, and it swam straightforwardly to the goal region (Supplementary Movie 2). In population data $(n=6)$, the percentage of correct choice was comparable to chance levels during the 'no shock' trials (49.4 \pm 9.4\%), then increased to $77.5 \pm 10.3 \%$ during the first 20 'shock' trials, and eventually achieved 93.3 $\pm 7.5 \%$ success in trials $61-80$ (Figure $2 \mathrm{~B}$ ). The latency to reach the goal region also decreased from $12.85 \pm 7.18 \mathrm{sec}$ (all 'no shock' trials) to $6.46 \pm 3.83 \mathrm{sec}$ (all 'shock' trials) (Figure 2C), clearly demonstrating the learning of avoidance behavior to the goal arm associated with a red color in this paradigm. We next examined whether the acquired memory is retained on the next day. Fish $(\mathrm{n}=5)$ were trained with the same protocol and the percentage of correct choice rate was increased from $51.9 \pm 7.3 \%$ at trial $1-20$ to $85.0 \pm 6.3 \%$ at trial $100-120$. Then, those learner fish were tested on the next day with the same apparatus in the absence of the electric shock. The correct choice rate in trial $1-20$ on the next day was kept high $(79.8 \pm 2.8 \%)$, clearly demonstrating that the acquired memory was retained for 24 hours (Figure 2D). 


\section{Discussion}

In our novel paradigm, zebrafish begin to show a significantly high success rate in learning avoidance within 20 trials of the task and the entire training procedure takes less than 2 hours. This is surprisingly rapid compared with similar behavioral paradigms (Darland and Dowling, 2001; Mueller and Neuhauss, 2012; Pradel et al., 1999), possibly because the visual cue presentation is on the floor and thus visible for the fish regardless of its orientation. It is also possible that the fish did not discriminate the color of the visual stimuli, but discriminated the contrast of the visual stimuli. In addition to the rapid learning process, our paradigm is fully automated and thus enables multiple experiments to be undertaken simultaneously. These advantages may dramatically increase the productivity of studies requiring large numbers of animals such as drug screening and mutant screening.

Active avoidance task, in which a fish has to escape into another region upon presentation of a cue to avoid an electric shock, is one of the most popular paradigms for examining cognitive ability in teleost. However, as naïve fish can also reach the other compartment during the cue presentation because of their inherent hyperlocomotivity, it is difficult to quantitatively evaluate the degree of learning in such active avoidance paradigms. In contrast, our paradigm showed a high degree of quantitative learning because the rate of correct choice in the naïve fish was approximately $50 \%$.

In this manuscript, we have attached all source codes and mat files as Supplementary toolbox 1 . Ymaze_discrimination.m $m$ is the source code for operating Y-maze discrimination task with the same protocol as the experiments described herein. Experimenters can also operate other types (plus-maze or shuttle-box) of paradigm or modify parameters such as time schedules and floor colors via GUI by opening the custom_maze.fig (Supplementary figure 1.). In addition, our MATLAB source code for position detection and visual cue presentation system is simple and easy to customize. This enables each experimenter to combine our system with various rules (e.g., various reward or punishment), various characteristics of visual cues (e.g., color (Avdesh et al., 2012), pattern, or specific object (Gerlai et al., 2009)), and various tank shapes to focus on their cognitive function of interest. Recently zebrafish has gained attention as a model animal for studying many cognitive functions such as emotion(Agetsuma et al., 2010), sensory perception(Yaksi et al., 2009), and motor learning(Ahrens et al., 2012). We ourselves also demonstrated that zebrafish long-term memory is easily tested by our paradigm. In addition, taking advantage of low cost, high throughput screening for behavioral phenotype, drug discovery with zebrafish has been succeeded(Rihel et al., 2010). Our 
system could provide a better way to both study cognitive behavior in zebrafish and conduct large population screening, thus enabling further insights for developing new therapies for human cognitive disease.

\section{Acknowledgement}

This work was supported in parts by Grant in Aid for Scientific Research (23120008, 23-9726) and Strategic Research Program for Brain Science from MEXT of Japan and CREST from JST, Japan.

\section{References}

Agetsuma, M., Aizawa, H., Aoki, T., Nakayama, R., Takahoko, M., Goto, M., Sassa, T., Amo, R., Shiraki, T., Kawakami, K., Hosoya, T., Higashijima, S., Okamoto, H., 2010. The habenula is crucial for experience-dependent modification of fear responses in zebrafish. Nat. Neurosci. 13, 1354-6.

Ahrens, M.B., Li, J.M., Orger, M.B., Robson, D.N., Schier, A.F., Engert, F., Portugues, R., 2012. Brain-wide neuronal dynamics during motor adaptation in zebrafish. Nature 485, 471-7.

Al-Imari, L., Gerlai, R., 2008. Sight of conspecifics as reward in associative learning in zebrafish (Danio rerio). Behav. Brain Res. 189, 216-9.

Aoki, T., Kinoshita, M., Aoki, R., Agetsuma, M., Aizawa, H., Yamazaki, M., Takahoko, M., Amo, R., Arata, A., Higashijima, S. ichi, Tsuboi, T., Okamoto, H., 2013. Imaging of neural ensemble for the retrieval of a learned behavioral program. Neuron 78, 881-894.

Avdesh, A., Martin-Iverson, M.T., Mondal, A., Chen, M., Askraba, S., Morgan, N., Lardelli, M., Groth, D.M., Verdile, G., Martins, R.N., 2012. Evaluation of color preference in zebrafish for learning and memory. J. Alzheimers. Dis. 28, 459-69.

Darland, T., Dowling, J.E., 2001. Behavioral screening for cocaine sensitivity in mutagenized zebrafish. Proc. Natl. Acad. Sci. U. S. A. 98, 11691-6.

Gerlai, R., Fernandes, Y., Pereira, T., 2009. Zebrafish (Danio rerio) responds to the animated image of a predator: towards the development of an automated aversive task. Behav. Brain Res. 201, 318-24.

Higashijima, S., Masino, M.A., Mandel, G., Fetcho, J.R., 2004. Engrailed-1 expression marks a primitive class of inhibitory spinal interneuron. J. Neurosci. 24, 5827-39.

Kawakami, K., Takeda, H., Kawakami, N., Kobayashi, M., Matsuda, N., Mishina, M., 2004. A transposon-mediated gene trap approach identifies developmentally regulated genes in zebrafish. Dev. Cell 7, 133-44. 
Lau, B., Bretaud, S., Huang, Y., Lin, E., Guo, S., 2006. Dissociation of food and opiate preference by a genetic mutation in zebrafish. Genes. Brain. Behav. 5, 497-505.

Levin, E.D., Chen, E., n.d. Nicotinic involvement in memory function in zebrafish. Neurotoxicol. Teratol. 26, 731-5.

Meng, X., Noyes, M.B., Zhu, L.J., Lawson, N.D., Wolfe, S.A., 2008. Targeted gene inactivation in zebrafish using engineered zinc-finger nucleases. Nat. Biotechnol. 26, 695-701.

Mueller, K.P., Neuhauss, S.C.F., 2012. Automated visual choice discrimination learning in zebrafish (Danio rerio). J. Integr. Neurosci. 11, 73-85.

Mueller, T., Wullimann, M.F., 2009. An evolutionary interpretation of teleostean forebrain anatomy. Brain. Behav. Evol. 74, 30-42.

Portavella, M., Torres, B., Salas, C., 2004. Avoidance response in goldfish: emotional and temporal involvement of medial and lateral telencephalic pallium. J. Neurosci. 24, 2335-42.

Pradel, G., Schachner, M., Schmidt, R., 1999. Inhibition of memory consolidation by antibodies against cell adhesion molecules after active avoidance conditioning in zebrafish. J. Neurobiol. 39, 197-206.

Rihel, J., Prober, D.A., Arvanites, A., Lam, K., Zimmerman, S., Jang, S., Haggarty, S.J., Kokel, D., Rubin, L.L., Peterson, R.T., Schier, A.F., 2010. Zebrafish behavioral profiling links drugs to biological targets and rest/wake regulation. Science 327, 348-51.

Rodríguez, F., López, J.C., Vargas, J.P., Gómez, Y., Broglio, C., Salas, C., 2002. Conservation of spatial memory function in the pallial forebrain of reptiles and ray-finned fishes. J. Neurosci. 22, 2894-903.

Sison, M., Gerlai, R., 2010. Associative learning in zebrafish (Danio rerio) in the plus maze. Behav. Brain Res. 207, 99-104.

Westerfield, M., 2007. The Zebrafish Book. A guide for the laboratory use of zebrafish (Danio rerio), 5th ed. Univ. of Oregon Press, Eugene.

Yaksi, E., von Saint Paul, F., Niessing, J., Bundschuh, S.T., Friedrich, R.W., 2009. Transformation of odor representations in target areas of the olfactory bulb. Nat. Neurosci. 12, 474-82. 


\section{Figure legends}

\section{Figure 1.}

(A) Schematic drawing of experimental setup. (B) Task Rule. Fish behavior is categorized into three types: Correct; fish first visited goal region, Incorrect; fish first visited non-goal region, Stay; fish visited neither goal region nor non-goal region throughout the trial.

\section{Figure 2.}

(A) Typical result in a single zebrafish. (top) The choice of the fish at each trial. (middle) Average learning curve of all 80 trials. Correct rate is the percentage of correct trials over 10 trials (bottom). Latency from the cue onset to reaching the goal region. Asterisks are trials in which the fish received the electric shock. (B) Mean correct rate of all fish $(n=6)$. Stay trials are excluded from the calculation so that the chance level is $50 \%$. Dotted line indicates the chance level $(50 \%)$. ${ }^{*} P<$ 0.001, paired t-test. Error bars represent the s.e.m. (C) Mean Latency of all fish $(n=6)$ in 'no shock' trials and 'shock' trials. ${ }^{*} P<0.05$, paired t-test. Error bars represent the s.e.m. (D) Long-term memory test. Mean correct rate of all fish $(n=5)$ at trial $1-20$, trial $100-120$, and trial $1-20$ on the next day (24 hr later). Dotted line indicates the chance level $(50 \%)$. $* P<0.05$, paired t-test. Error bars represent the s.e.m. 
Figures $\quad$ Figure 1 A
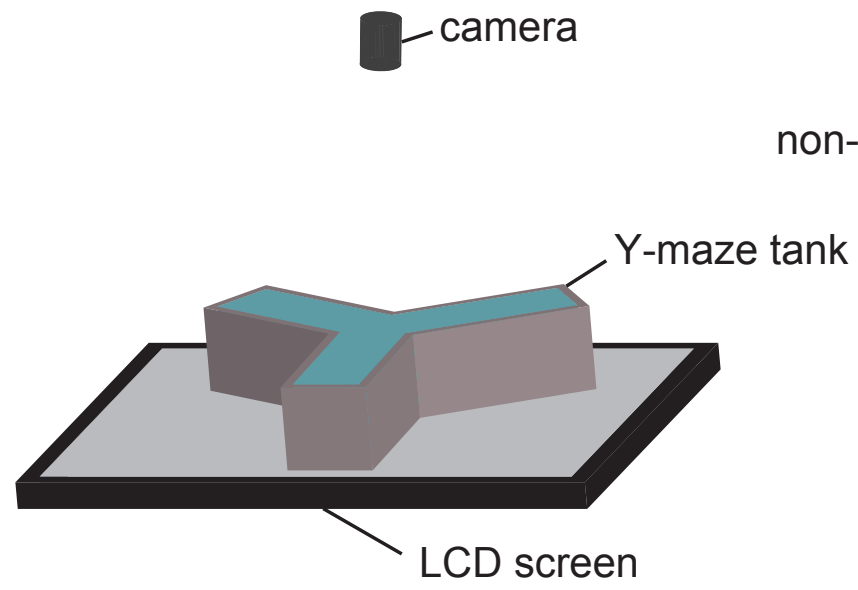

B

correct

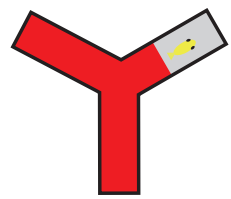

$\gamma$

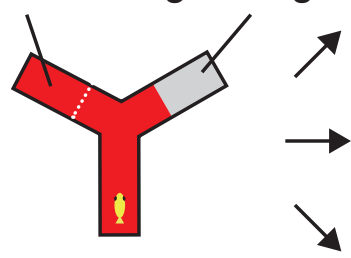

incorrect

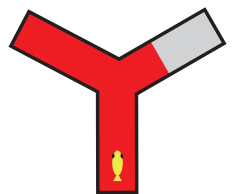




\section{A Figure 2}

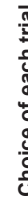

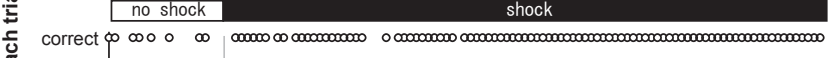
용<smiles>[O]</smiles>

o incorrect

임

stay

సँّ

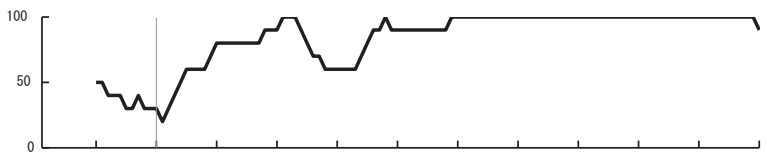

ब઼

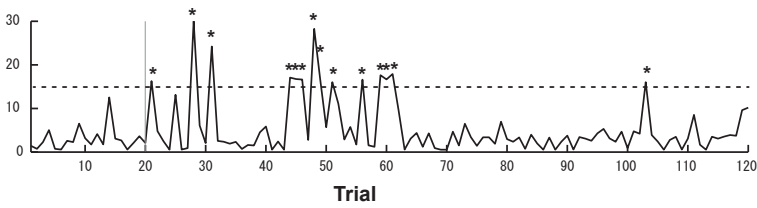

B

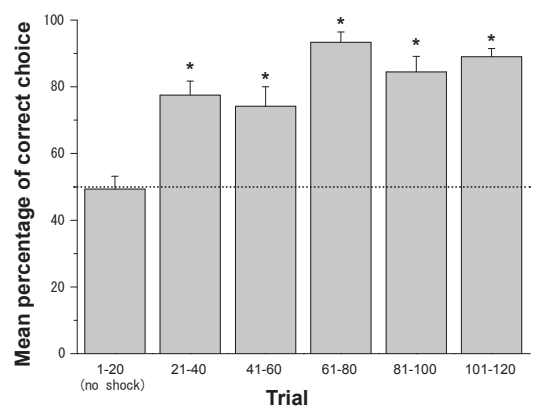

C

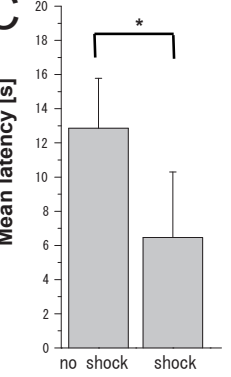

D

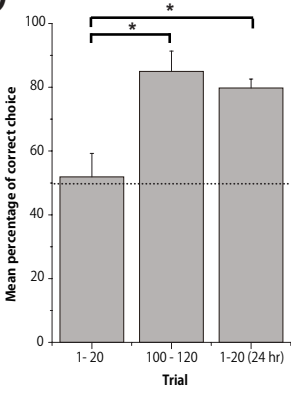

